\title{
TESTING THE EVOLUTIONARY SEQUENCE OF HIGH-MASS PROTOSTARS WITH CARMA
}

\author{
SCOTT SCHNEE AND John M. CARPENTER \\ Department of Astronomy, California Institute of Technology, MC 105-24 Pasadena, CA 91125, USA; schnee@ astro.caltech.edu \\ Received 2008 December 19; accepted 2009 April 15; published 2009 June 1
}

\begin{abstract}
We present 1" resolution Combined Array for Research in Millimeter-wave Astronomy (CARMA) observations

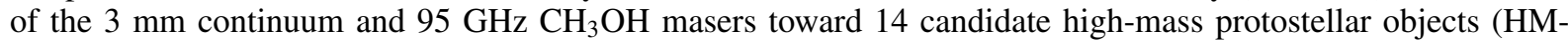
POs). Dust continuum emission is detected toward seven HMPOs, and $\mathrm{CH}_{3} \mathrm{OH}$ masers toward five sources. The $3 \mathrm{~mm}$ continuum sources have diameters $<2 \times 10^{4} \mathrm{AU}$, masses between 21 and $1200 M_{\odot}$, and volume densities $>10^{8} \mathrm{~cm}^{-3}$. Most of the $3 \mathrm{~mm}$ continuum sources are spatially coincident with compact $\mathrm{H}$ II regions and/or water masers, and are presumed to be formation sites of massive stars. A strong correlation exists between the presence

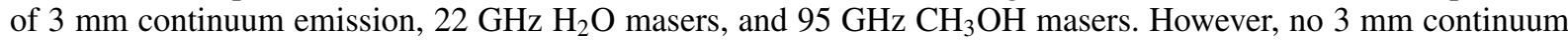
emission is detected toward ultracompact $\mathrm{H}$ II regions lacking maser emission. These results are consistent with the hypothesis that $22 \mathrm{GHz} \mathrm{H} \mathrm{H}_{2} \mathrm{O}$ masers and $\mathrm{CH}_{3} \mathrm{OH}$ masers are signposts of an early phase in the evolution of an HMPO before an expanding $\mathrm{H}$ II region destroys the accretion disk.
\end{abstract}

Key words: masers - stars: formation

Online-only material: color figures

\section{INTRODUCTION}

The evolutionary sequence of low-mass star formation is broadly understood. Gravity in a prestellar core eventually overcomes the thermal, turbulent, magnetic, and rotational support to form a protostar. This protostar is surrounded by an envelope and accretion disk, and launches a bipolar outflow. As the protostar accretes mass, the surrounding envelope and disk are cleared by the winds and outflows until no more mass is left to accrete (see, e.g., McKee \& Ostriker 2007).

High-mass protostars share many of the same observational characteristics as their low-mass counterparts: outflows (Zhang et al. 2001), jets (Caratti O Garatti et al. 2008), envelopes (Molinari et al. 1996), and possibly disks (Cesaroni et al. 2005). However, the elevated radiation environment that accompanies high-mass stars raises the question as to what degree highmass star formation is merely a scaled version of low-mass star formation. Answering that question is not straightforward, partly because high-mass star-forming regions are less common than the low-mass counterparts, and tend to be more distant. The greater distance, coupled with the propensity of highmass protostellar objects (HMPOs) to form in clusters and the disruptive influence of jets, outflows and $\mathrm{H}$ II regions, complicates interpretation of the observations (Zinnecker \& Yorke 2007). Therefore, to study the initial conditions of high-mass star formation, it is important to identify young HMPOs before their surroundings have been altered by energetic processes.

Surveys for high-mass protostars have been conducted over the years at a variety of wavelengths. Optical and radio continuum surveys of the galactic plane have identified $\mathrm{H}$ II regions in which massive star formation is already well underway (e.g., Maršálková 1974; Altenhoff et al. 1979; Haynes et al. 1979). Wood \& Churchwell (1989) used the IRAS catalog to search for far-infrared (FIR) sources associated with ultracompact H II (UCH II) regions and high-mass protostars embedded in dense cores. Massive star formation is thought to be associated with infrared dark clouds (IRDCs), which are seen in absorption against the diffuse mid-infrared background of the galactic plane
(Perault et al. 1996; Egan et al. 1998). The masses of IRDCs suggest that they may form stellar clusters (Rathborne et al. 2006, 2007), and IRDCs are known to host both protostars and starless cores (Wang et al. 2006). More recently, surveys with the Spitzer Space Telescope such as GLIMPSE (Benjamin et al. 2003) have identified HMPOs based on mid-infrared colors (Kumar \& Grave 2007; Cyganowski et al. 2008).

High-mass star formation also coincides with a variety of masers, as demonstrated by targeted surveys of known HMPOs and blind surveys of the galactic plane (e.g., Szymczak et al. 2000; Pandian et al. 2007). Interferometric observations have shown that water masers (at $22.2 \mathrm{GHz}$ ) are associated with Jshocks from jets and winds ejected by massive young stellar objects (Torrelles et al. 2003; Goddi et al. 2005; Moscadelli et al. 2005). Methanol masers (at $6.7 \mathrm{GHz}$ ) are less understood, but are thought to trace the shock where a jet meets the ambient circumstellar gas (De Buizer 2003) and/or the innermost portions of a disk around a protostar (Edris et al. 2005). Hydroxyl maser emission (at $1.7 \mathrm{GHz}$ ) may also come from a disk around an accreting protostar (Edris et al. 2005), and is also associated with both H II and UCH II regions (Edris et al. 2007).

Although there are many tracers of high-mass star formation (masers, radio continuum, infrared objects), they are not always spatially coincident or even found within the same object. Over the years, various observations of HMPOs have been unified under a common evolutionary sequence (see reviews by Garay \& Lizano 1999; Menten et al. 2005; Beuther et al. 2007; Zinnecker \& Yorke 2007). IRDCs are thought to host the youngest protostars, perhaps surrounded by accretion disks and driving jets and outflows. The disk, jet, and outflow system are conducive for the formation of $\mathrm{H}_{2} \mathrm{O}, \mathrm{CH}_{3} \mathrm{OH}$, and possibly $\mathrm{OH}$ maser emission. This evolutionary stage is also distinguished by dust emission from the disk and envelope around the protostar. The development of an $\mathrm{H}$ II region is suppressed initially by the continued accretion of material onto the star (Walmsley 1995). As the protostar increases in mass, temperature, and luminosity, an $\mathrm{H}$ II region forms that is initially gravitationally bound to the star and remains compact in size (Keto 2002). Eventually the protostar becomes massive enough to produce an $\mathrm{H}$ II region 
Table 1

Source Properties

\begin{tabular}{|c|c|c|c|c|c|c|c|}
\hline IRAS Name & Group $^{a}$ & $\begin{array}{l}\text { Distance }^{b} \\
(\mathrm{kpc})\end{array}$ & $\begin{array}{c}V_{\mathrm{LSR}}^{\mathrm{b}} \\
\left(\mathrm{km} \mathrm{s}^{-1}\right)\end{array}$ & $\log \left(L_{\text {total }} / L_{\odot}\right)^{\mathrm{b}}$ & $\begin{array}{l}\text { Peak } S_{v}(1.2 \mathrm{~mm})^{\mathrm{c}} \\
\left(\mathrm{mJy} \mathrm{beam}^{-1}\right)\end{array}$ & $\begin{array}{c}\text { Integrated } S_{\nu}(1.2 \mathrm{~mm})^{\mathrm{c}} \\
(\mathrm{Jy})\end{array}$ & $\begin{array}{c}\text { Core Mass }^{\mathrm{d}} \\
\left(M_{\odot}\right)\end{array}$ \\
\hline 18345-0641 & 1 & 9.5 & 95.9 & 4.6 & 265 & 1.4 & 6860 \\
\hline 18440-0148 & 1 & 8.3 & 97.6 & 4.7 & 120 & 0.5 & 1717 \\
\hline $18517+0437$ & 1 & 2.9 & 43.9 & 4.1 & 812 & 7.2 & 2310 \\
\hline $18566+0408$ & 1 & 6.7 & 85.2 & 4.8 & 486 & 1.6 & 2110 \\
\hline $23151+5912$ & 1 & 5.7 & -54.4 & 5.0 & 406 & 2.0 & 1229 \\
\hline $19217+1651$ & 2 & 10.5 & 3.5 & 4.9 & 640 & 2.6 & 9518 \\
\hline $20126+4104$ & 2 & 1.7 & -3.8 & 3.9 & 1087 & 5.8 & 460 \\
\hline $20332+4124$ & 2 & 3.9 & -2.0 & 4.4 & 265 & 3.5 & 1529 \\
\hline $23033+5951$ & 2 & 3.5 & -53.1 & 4.0 & 631 & 3.5 & 2327 \\
\hline $23139+5939$ & 2 & 4.8 & -44.7 & 4.4 & 530 & 2.3 & 1759 \\
\hline $19220+1432$ & 3 & 5.5 & 68.8 & 4.4 & 256 & 1.9 & 3406 \\
\hline $20205+3948$ & 3 & 4.5 & -1.7 & 4.5 & 104 & 0.9 & 548 \\
\hline $22134+5834$ & 3 & 2.6 & -18.3 & 4.1 & 229 & 2.5 & 436 \\
\hline $22570+5912$ & 3 & 5.1 & -46.7 & 4.7 & 215 & 2.1 & 1469 \\
\hline
\end{tabular}

Notes.

${ }^{a}$ Group 1: with $22 \mathrm{GHz} \mathrm{H}_{2} \mathrm{O}$ and/or $6.7 \mathrm{CH}_{3} \mathrm{OH}$ masers and no centimeter continuum; Group 2: with $22 \mathrm{GHz}_{2} \mathrm{O}$ and/or $6.7 \mathrm{CH} 3 \mathrm{OH}$ masers and centimeter continuum; Group 3: without $22 \mathrm{GHz} \mathrm{H} \mathrm{H}_{2} \mathrm{O}$ or $6.7 \mathrm{CH}_{3} \mathrm{OH}$ masers and with centimeter continuum.

b From Sridharan et al. (2002).

c $1.2 \mathrm{~mm}$ continuum flux densities from Beuther et al. (2002a), measured in a $11^{\prime \prime}$ beam.

${ }^{\mathrm{d}}$ Mass of gas and dust inferred from $1.2 \mathrm{~mm}$ continuum flux densities, taken from Beuther et al. (2002a).

that escapes the stellar gravitational potential (Keto 2007). The $\mathrm{H}$ II region expands to engulf the accretion disk and envelope, which destroys the source of the masers, jets, and outflows (Lo et al. 1975; Genzel \& Downes 1977; Codella \& Felli 1995; Codella et al. 2004). This evolutionary sequence has provided a framework to explain a multitude of observations (e.g., Molinari et al. 1996; Beuther et al. 2002b; Minier et al. 2005; van der Tak \& Menten 2005).

An observational prediction of this evolutionary sequence is that compact dust continuum emission from an accretion disk or envelope will be present in HMPOs containing maser emission (Codella et al. 2004). At a more advanced stage when the $\mathrm{H}$ II region has grown, maser emission will be suppressed and dust emission from the disk and envelope should be at a much reduced level. Single-dish maps of the dust emission exist for a number of HMPOs (Beuther et al. 2002a), but no clear correlation is present between the dust continuum luminosity and the presence of maser emission. However, the resolution of these observations $\left(11^{\prime \prime}\right)$ is insufficient to distinguish compact emission around HMPOs from the ambient dense core. Interferometric observations have achieved significantly higher resolution $\left(\sim 5^{\prime \prime}\right)$, but they cover a limited range of presumed evolutionary states and are insufficient to test the proposed evolutionary sequence (Molinari et al. 2002; Beltrán et al. 2004; Cesaroni et al. 2005; Zhang et al. 2007; Reid \& Matthews 2008).

In this paper, we present high resolution (1") Combined Array for Research in Millimeter-wave Astronomy (CARMA) observations of the dust continuum and $95 \mathrm{GHz} \mathrm{CH}_{3} \mathrm{OH}$ maser emission toward 14 HMPOs to test the evolutionary sequence for massive stars described above. The HMPO sample was selected to cover the primary evolutionary stages in this model. With the order of magnitude improvement in angular resolution over previous single-dish surveys, we can isolate any compact dust emission originating from a disk or envelope associated with an HMPO. In Section 2, we describe the sample of HMPOs in our survey, and the available ancillary information regarding their evolutionary state. The new CARMA observations are described in Section 3 and the observational results are presented in Section 4. In Section 5, we compare our results with the proposed evolutionary sequence for massive stars.

\section{SAMPLE}

We drew a sample of 14 high-mass protostellar candidates from the survey of Sridharan et al. (2002). The HMPOs in the Sridharan et al. (2002) list have IRAS colors of UCH II regions (Wood \& Churchwell 1989), are detected in the CS $J=2-1$ survey of Bronfman et al. (1996), are bright at FIR wavelengths $\left(F_{60}>90 \mathrm{Jy}, F_{100}>500 \mathrm{Jy}\right)$, are north of $-20^{\circ}$ declination, and are not detected above $25 \mathrm{mJy}$ in the $5 \mathrm{GHz}$ radio continuum surveys of (Gregory \& Condon 1991; Wright et al. 1994; Griffith et al. 1994).

The properties of the HMPOs selected for this study are summarized in Table 1. The HMPOs have a bolometric luminosity between $10^{3.9}$ and $10^{5.0} L_{\odot}$. The distances to the sources range from 1.7 to $10.5 \mathrm{kpc}$, where the near-far distance ambiguity has been resolved through a variety of methods as described in Sridharan et al. (2002). The dense cores associated with the HMPOs have masses of $\sim 400-10,000 M_{\odot}$ contained within a $\sim 1 \mathrm{pc}$ region. We adopted the velocity and association with centimeter continuum emission, $\mathrm{H}_{2} \mathrm{O}$ masers and $\mathrm{CH}_{3} \mathrm{OH}$ masers for each source given in Sridharan et al. (2002). The $3.6 \mathrm{~cm}$ radio continuum emission was observed with the Very Large Array $(\mathrm{VLA})^{1}$ in B-array with a sensitivity of $\sigma \sim 1 \mathrm{mJy}$, and the coordinates of the detections were kindly provided by $\mathrm{H}$. Beuther (2008, private communication). The $1.2 \mathrm{~mm}$ continuum fluxes and positions were measured with MAMBO on the IRAM $30 \mathrm{~m}$ telescope at a resolution of $11^{\prime \prime}$ (Beuther et al. 2002a). The association with $\mathrm{OH}$ masers is presented in Edris et al. (2007), and were detected with the Nancay radio telescope and the Robert C. Byrd Green Bank Telescope (GBT).

\footnotetext{
1 The VLA is operated by the National Radio Astronomy Observatory, which is a facility of the National Science Foundation, operated under cooperative agreement by Associated Universities, Inc.
} 
Table 2

CARMA Observing Parameters

\begin{tabular}{|c|c|c|c|c|c|c|}
\hline IRAS Name & $\begin{array}{l}\text { R.A. }^{a} \\
\text { J2000 }\end{array}$ & $\begin{array}{l}\text { Decl. }^{\mathrm{a}} \\
\mathrm{J} 2000\end{array}$ & Phase Calibrators & $\begin{array}{c}\text { Resolution }{ }^{\mathrm{b}} \\
\left({ }^{\prime \prime} \times{ }^{\prime \prime}\right) \\
\end{array}$ & $\begin{array}{c}\text { Continuum Noise } \\
\left(\mathrm{mJy}_{\text {beam }}{ }^{-1}\right)\end{array}$ & $\begin{array}{c}\text { Noise/Channel }^{\mathrm{c}} \\
\left(\mathrm{mJy} \mathrm{beam}^{-1}\right)\end{array}$ \\
\hline 18345-0641 & $18: 37: 17.02$ & $-06: 38: 30.70$ & $1751+096,1743-038$ & $1.6 \times 0.7$ & 1.3 & 62 \\
\hline $18440-0148$ & $18: 46: 36.56$ & $-01: 45: 21.20$ & $1751+096,1743-038$ & $1.6 \times 0.7$ & 0.9 & 34 \\
\hline $18517+0437$ & $18: 54: 14.32$ & $+04: 41: 39.69$ & $1751+096,1743-038$ & $1.4 \times 0.7$ & 1.2 & 49 \\
\hline $18566+0408$ & 18:59:10.02 & $+04: 12: 14.69$ & $1751+096,1743-038$ & $1.6 \times 0.7$ & 0.9 & 26 \\
\hline $19217+1651$ & $19: 23: 58.77$ & $+16: 57: 44.80$ & $1751+096,1925+211$ & $1.5 \times 0.7$ & 1.2 & 31 \\
\hline $19220+1432$ & $19: 24: 20.05$ & $+14: 38: 03.60$ & $1751+096,1925+211$ & $1.4 \times 0.7$ & 1.0 & 32 \\
\hline $20126+4104$ & $20: 14: 25.86$ & $+41: 13: 33.99$ & BLLAC,2007+404 & $1.4 \times 0.8$ & 1.0 & 37 \\
\hline $20205+3948$ & $20: 22: 20.87$ & $+39: 58: 15.00$ & BLLAC,2007+404 & $1.6 \times 0.8$ & 1.5 & 78 \\
\hline $20332+4124$ & $20: 34: 59.72$ & $+41: 34: 49.40$ & BLLAC,2007+404 & $1.2 \times 0.6$ & 1.0 & 39 \\
\hline $22134+5834$ & $22: 15: 09.51$ & $+58: 49: 05.99$ & BLLAC,2007+404 & $1.5 \times 0.8$ & 1.3 & 61 \\
\hline $22570+5912$ & $22: 59: 05.37$ & $+59: 28: 19.18$ & BLLAC,0102+584 & $1.0 \times 0.8$ & 0.6 & 30 \\
\hline $23033+5951$ & $23: 05: 25.31$ & $+60: 08: 06.28$ & BLLAC,0102+584 & $1.0 \times 0.8$ & 0.6 & 22 \\
\hline $23139+5939$ & $23: 16: 10.45$ & $+59: 55: 28.48$ & BLLAC,0102+584 & $1.0 \times 0.8$ & 0.6 & 26 \\
\hline $23151+5912$ & $23: 17: 21.02$ & $+59: 28: 48.48$ & BLLAC,0102+584 & $1.2 \times 0.7$ & 0.8 & 34 \\
\hline
\end{tabular}

Notes.

a Phase center positions taken from $1.2 \mathrm{~mm}$ coordinates listed in Beuther et al. (2002a).

${ }^{\mathrm{b}}$ FWHM synthesized beam size with natural weighting.

${ }^{\mathrm{c}}$ Channel width of $0.488 \mathrm{MHz}$.

The 14 HMPOs that we observed were divided into three categories depending on the presence or absence of centimeter continuum emission indicative of an $\mathrm{UCH}$ II region, $6.7 \mathrm{GHz}$ $\mathrm{CH}_{3} \mathrm{OH}$ masers, or $22 \mathrm{GHz} \mathrm{H}_{2} \mathrm{O}$ masers. In order of increasing age according to the proposed evolutionary sequence (Lo et al. 1975; Genzel \& Downes 1977; Codella \& Felli 1995), our sample contains: (1) five sources with $\left(\mathrm{CH}_{3} \mathrm{OH}\right.$ or $\left.\mathrm{H}_{2} \mathrm{O}\right)$ maser emission but no centimeter continuum, (2) five sources with both centimeter continuum and maser emission, and (3) four sources with centimeter continuum but no maser emission. For each of these three categories, the range of distances (2.9-9.5 kpc, $1.7-10.5 \mathrm{kpc}, 2.6-5.5 \mathrm{kpc}$, respectively) and luminosities $\left(10^{4.1}-10^{5.0} L_{\odot}, 10^{3.9}-10^{4.9} L_{\odot}, 10^{4.1}-10^{4.7} L_{\odot}\right)$ sampled are similar. We centered each CARMA map on the position of the peak $1.2 \mathrm{~mm}$ continuum emission derived from $11^{\prime \prime}$ resolution single-dish observations (Beuther et al. 2002a). Note that in Sridharan et al. (2002) the source IRAS 183450641 is associated with both maser and centimeter continuum emission. For the purposes of this survey, we consider IRAS $18345-0641$ to be a maser-only HMPO because the masers and $1.2 \mathrm{~mm}$ continuum peak are coincident to within $1^{\prime \prime}$, but the centimeter continuum emission is offset from the maser position by $44^{\prime \prime}$, or 2 pc.

\section{CARMA OBSERVATIONS}

Continuum and spectral line observations in the $3 \mathrm{~mm}$ window were obtained with CARMA. CARMA is a 15 element interferometer consisting of nine 6.1 meter antennas and six 10.4 meter antennas. Data were taken in the CARMA Barray configuration between 2007 December 24 and 2008 February 11 . The projected baselines in this configuration range from $65 \mathrm{~m}$ to $800 \mathrm{~m}$. The CARMA correlator records signals in three separate bands, each with an upper and lower sideband. Two bands were configured for maximum bandwidth $(468 \mathrm{MHz}$ with 15 channels per band) to observe continuum emission, providing a total continuum bandwidth of $1.87 \mathrm{GHz}$. One band was configured with $31 \mathrm{MHz}$ bandwidth across 63 channels (with a resolution of $0.488 \mathrm{MHz}$ or $1.6 \mathrm{~km} \mathrm{~s}^{-1}$ per channel) to observe the hyperfine lines of $\mathrm{CH}_{3} \mathrm{CN}$ (5-4) in the lower sideband (from 92.959 to $92.987 \mathrm{GHz}$ ) and $\mathrm{CH}_{3} \mathrm{OH}\left(8_{0,8^{-}}\right.$
$7_{1,7}$ ) in the upper sideband (at $95.2 \mathrm{GHz}$ ). The FWHM of the synthesized beam with natural weighting of the visibilities is $\sim 1^{\prime \prime}$ (see Table 2), and the largest angular scale that can be accurately imaged in the maps is $\sim 5^{\prime \prime}$. The half-power beam width of the $10.4 \mathrm{~m}$ antennas is $73^{\prime \prime}$ at the observed frequencies.

The observing sequence was to integrate on a primary phase calibrator for 2.5 minutes, an HMPO for 7 minutes, and a secondary phase calibrator for 2.5 minutes. This cycle was repeated for $\sim 3 \mathrm{hr}$ for each HMPO. Calibration and imaging were done using the MIRIAD data reduction package (Sault et al. 1995). The sources observed, along with the phase center, calibrators and the noise in the resultant continuum maps and spectra are listed in Table 2 . The secondary calibrator was used to test the accuracy of the phase transfer from the primary calibrator. A passband calibrator (3C454.3) was observed for 15 minutes in each set of observations, and radio pointing was performed every $2 \mathrm{hr}$ thereafter. Absolute flux calibration was accomplished using Neptune, Uranus, and MWC349 as primary flux calibrators and $3 \mathrm{C} 454.3$ as a secondary flux calibrator. Based on the repeatability of the quasar fluxes, the estimated random uncertainty in the measured source fluxes is $\sigma \sim 5 \%$. Because we spent only $\sim 3 \mathrm{hr}$ on each of the 14 HMPOs, we had sufficient signal to noise to detect $\mathrm{CH}_{3} \mathrm{CN}$ in only one source, IRAS $20126+4104$, and the $\mathrm{CH}_{3} \mathrm{CN}$ observations are not discussed further.

\section{RESULTS}

Here, we present the CARMA $3 \mathrm{~mm}$ continuum and $95 \mathrm{GHz}$ $\mathrm{CH}_{3} \mathrm{OH}$ maps and compare them with maps of $6.7 \mathrm{GHz} \mathrm{CH}_{3} \mathrm{OH}$

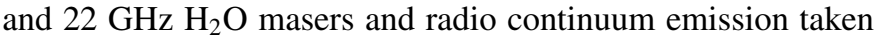
from the literature. We derive masses for the $3 \mathrm{~mm}$ detections, and present evidence for outflows emanating from these sources.

\section{1. $3 \mathrm{~mm}$ Continuиm}

We detect $3 \mathrm{~mm}$ continuum emission toward seven of the 14 HMPOs at the $3 \sigma$ level or greater. Contour plots of the $3 \mathrm{~mm}$ flux density for the detections are presented in Figure 1, and plots of the nondetections are shown in Figure 2. The sources IRAS $23033+5951$ and IRAS $19217+1651$ are resolved into 

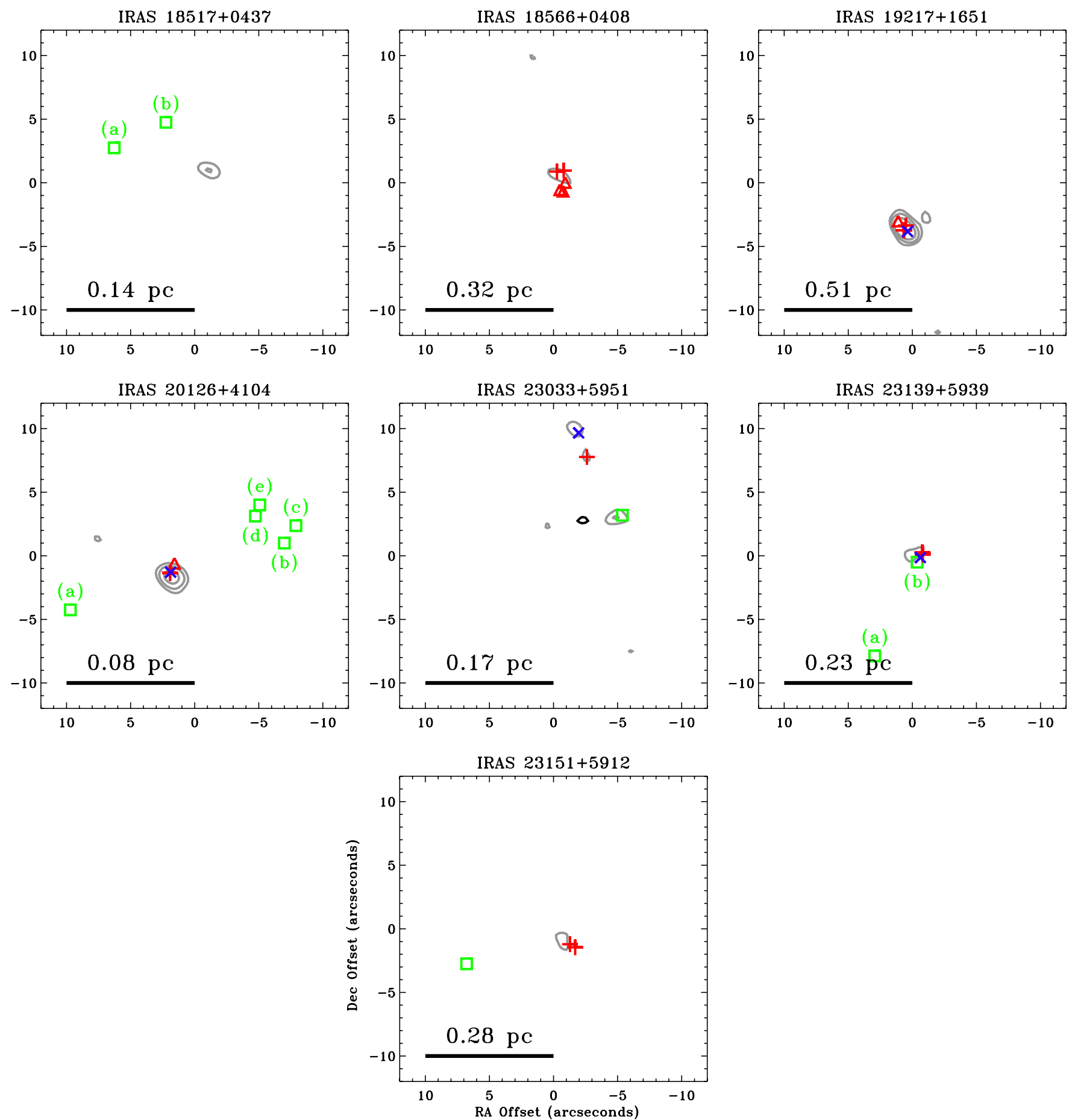

Figure 1. Contour plots of the $3 \mathrm{~mm}$ continuum flux density for HMPO candidates sources detected with CARMA at the $\geqslant 3 \sigma$ level, centered at the position of the peak 1.2 mm MAMBO (11" resolution) emission. Grey contours show emission at 3,6, and 12 times the noise in each map, and black contours show emission at

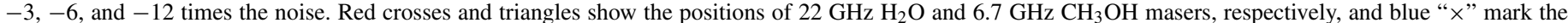
positions of 3.6 centimeter continuum sources (Beuther et al. 2002b; Sridharan et al. 2002, H. Beuther (2008, private communication). The source IRAS $18517+0437$ was not observed by Beuther et al. (2002b). Green squares show the $95 \mathrm{GHz} \mathrm{CH}_{3} \mathrm{OH}$ maser emission detected by CARMA. The resolution of the $3 \mathrm{~mm}$ continuum images is $\sim 1^{\prime \prime}$.

(A color version of this figure is available in the online journal.)

three and two components, respectively. None of the detected sources are more than a factor of $\sim 2$ larger than the $\sim 1^{\prime \prime}$ beam of our maps. The secondary phase calibrators, assumed to be point sources, have the same range of sizes as the detected HMPOs, so all of the $3 \mathrm{~mm}$ detections are consistent with point sources at $1^{\prime \prime}$ resolution blurred by seeing effects. The CARMA observations are sensitive to angular scales as large as $5^{\prime \prime}$, and resolve out the majority of the extended emission associated with the starforming regions seen in the $1.2 \mathrm{~mm}$ single-dish maps (Beuther et al. 2002a).

The positions and measured flux densities of the $3 \mathrm{~mm}$ continuum sources are listed in Table 3. The brightest $3 \mathrm{~mm}$ continuum source detected in each map is located within $6^{\prime \prime}$ of the centroid of the larger-scale core traced by single-dish 

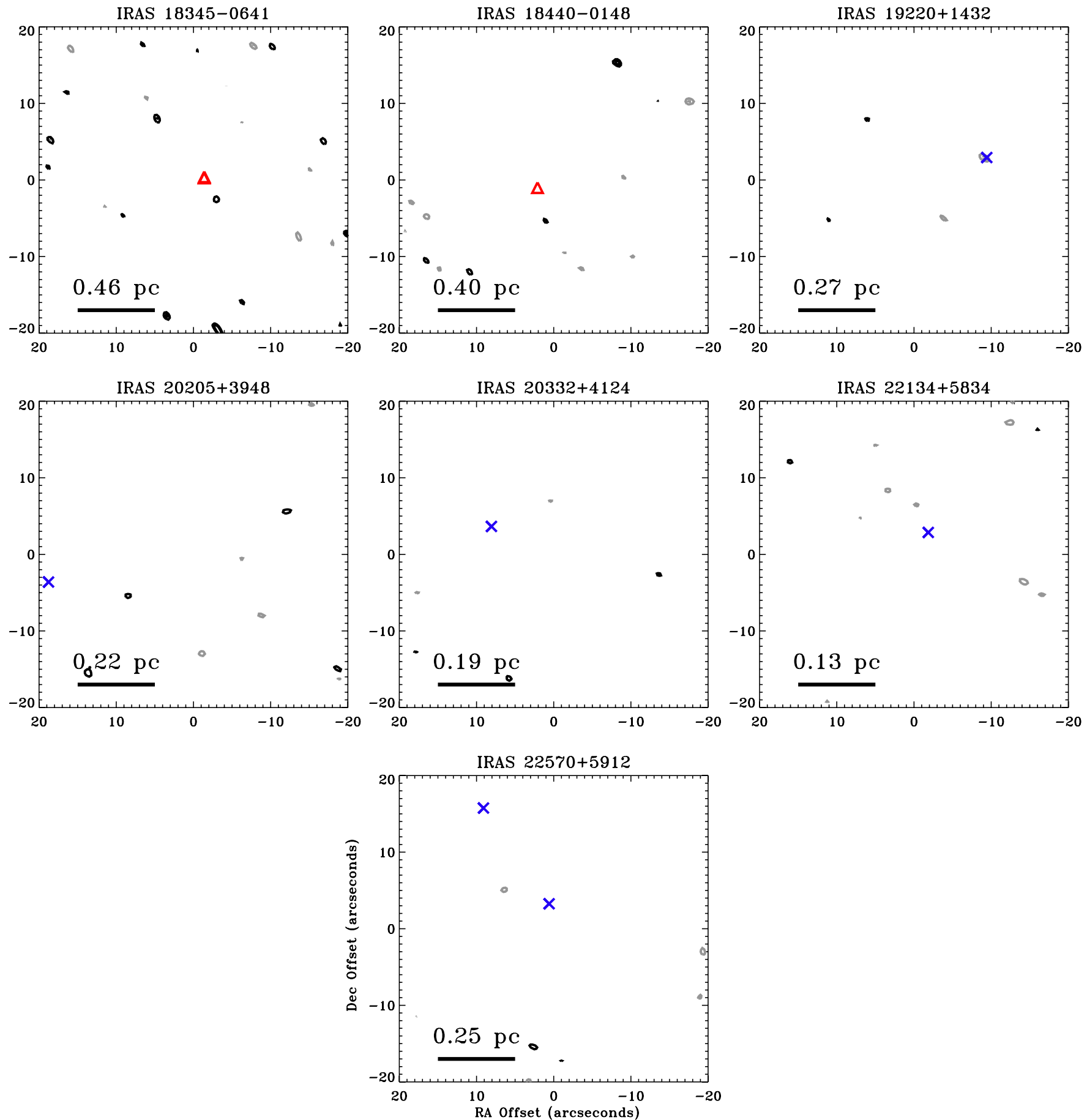

Figure 2. Contour plots of the $3 \mathrm{~mm}$ continuum flux density for eight HMPO candidates not detected with CARMA. Gray contours show fluxes at 2.0 and 2.5 times the noise in each map, and black contours show fluxes -2.5 and -2.0 times the noise. The symbols are otherwise the same as presented in Figure 1 .

(A color version of this figure is available in the online journal.)

$1.2 \mathrm{~mm}$ continuum emission as measured by Beuther et al. (2002a). Given the $11^{\prime \prime}$ resolution of the $1.2 \mathrm{~mm}$ observations and the difficulty in finding the exact center of nonsymmetric intensity distributions, the offsets between the $3 \mathrm{~mm}$ and $1.2 \mathrm{~mm}$ positions are most likely not significant.

\subsection{Masers}

We detect $95 \mathrm{GHz} \mathrm{CH}_{3} \mathrm{OH}$ emission toward five HMPOs. Two of the sources (IRAS 23033+5951 and IRAS 23151+5912) have a single $\mathrm{CH}_{3} \mathrm{OH}$ emission detection, and three (IRAS 18517+0437, IRAS 20126+4104, and IRAS 23139+5939) have multiple detections. Spectra for each of the detections are shown in Figure 3, and spectral parameters are summarized in Table 4. The $95 \mathrm{GHz} \mathrm{CH}_{3} \mathrm{OH}$ lines are bright (with brightness temperatures around a few hundred Kelvin), narrow (unresolved at $1.6 \mathrm{~km} \mathrm{~s}^{-1}$ resolution), and spatially unresolved. Therefore, the inferred brightness temperatures are lower limits and the lines are likely to be masing.

The five HMPOs that have $95 \mathrm{GHz} \mathrm{CH}_{3} \mathrm{OH}$ masers are also detected in the $3 \mathrm{~mm}$ continuum. The location of the presumed masers are marked on the $3 \mathrm{~mm}$ contour maps in Figure 1. Two $95 \mathrm{GHz} \mathrm{CH}_{3} \mathrm{OH}$ masers are coincident with the $3 \mathrm{~mm}$ 
Table 3

Properties of Detected $3 \mathrm{~mm}$ Continuum Sources

\begin{tabular}{|c|c|c|c|c|c|c|c|c|}
\hline IRAS Name & $\begin{array}{l}\text { R.A. } \\
\text { J2000 }\end{array}$ & $\begin{array}{l}\text { Decl. } \\
\text { J2000 }\end{array}$ & $\begin{array}{c}(\Delta \alpha, \Delta \delta)^{\mathrm{a}} \\
\left({ }^{\prime \prime},{ }^{\prime \prime}\right)\end{array}$ & $\begin{array}{c}S_{\text {peak }} \\
\left(\mathrm{mJy} \mathrm{beam}^{-1}\right)\end{array}$ & $\begin{array}{c}S_{\text {int }} \\
(\mathrm{mJy})\end{array}$ & $f_{d}^{\mathrm{b}}$ & $\begin{array}{l}\text { Mass } \\
\left(M_{\odot}\right)\end{array}$ & $\begin{array}{c}\text { Density } \\
\left(10^{8} \mathrm{~cm}^{-3}\right)\end{array}$ \\
\hline $18517+0437$ & $18: 54: 14.24$ & $+04: 41: 40.7$ & $(-1.1,1.0)$ & $8.5 \pm 1.4$ & $16 \pm 2$ & 1.0 & 74 & 8.6 \\
\hline $18566+0408$ & 18:59:09.99 & $+04: 12: 15.3$ & $(-0.4,0.6)$ & $4.4 \pm 1.2$ & $10 \pm 2$ & 1.0 & 250 & 2.3 \\
\hline $19217+1651$ & $19: 23: 58.81$ & $+16: 57: 41.1$ & $(0.6,-3.7)$ & $55 \pm 3$ & $66 \pm 1$ & 0.3 & 1200 & 2.9 \\
\hline $19217+1651$ & $19: 23: 58.70$ & $+16: 57: 42.0$ & $(-1.1,-2.8)$ & $4.9 \pm 1.3$ & $9 \pm 2$ & 1.0 & 540 & 1.3 \\
\hline $20126+4104$ & $20: 14: 26.03$ & $+41: 13: 32.6$ & $(1.8,-1.4)$ & $18 \pm 1$ & $33 \pm 2$ & 0.9 & 47 & 27 \\
\hline $23033+5951$ & $23: 05: 24.65$ & $+60: 08: 09.3$ & $(-4.9,3.0)$ & $4.4 \pm 0.9$ & $8 \pm 1$ & 1.0 & 54 & 3.6 \\
\hline $23033+5951$ & $23: 05: 24.97$ & $+60: 08: 14.2$ & $(-2.5,7.9)$ & $2.3 \pm 0.6$ & $6 \pm 1$ & 1.0 & 40 & 2.7 \\
\hline $23033+5951$ & 23:05:25.09 & $+60: 08: 16.3$ & $(-1.7,10.0)$ & $3.4 \pm 0.9$ & $8 \pm 1$ & 0.4 & 21 & 1.4 \\
\hline $23139+5939$ & $23: 16: 10.45$ & $+59: 55: 28.6$ & $(0.0,0.1)$ & $2.8 \pm 0.5$ & $12 \pm 2$ & 0.7 & 110 & 2.7 \\
\hline $23151+5912$ & $23: 17: 20.94$ & $+59: 28: 47.6$ & $(-0.8,-0.9)$ & $3.5 \pm 1.2$ & $8 \pm 2$ & 1.0 & 140 & 2.2 \\
\hline
\end{tabular}

Notes.

a Difference between the measured $3 \mathrm{~mm}$ continuum source and the $1.2 \mathrm{~mm}$ core (see Table 1 ).

${ }^{\mathrm{b}}$ Fraction of $3 \mathrm{~mm}$ emission from dust (see Section 4).

Table 4

Properties of Detected $95 \mathrm{GHz} \mathrm{CH}_{3} \mathrm{OH}$ Spectral Lines

\begin{tabular}{|c|c|c|c|c|c|c|}
\hline IRAS Name & $\begin{array}{l}\text { R.A. } \\
\text { J2000 }\end{array}$ & $\begin{array}{l}\text { Decl. } \\
\text { J2000 }\end{array}$ & $\begin{array}{c}\text { Separation }^{\mathrm{a}} \\
\left(10^{4} \mathrm{AU}\right)\end{array}$ & $\begin{array}{c}S_{\text {peak }} \\
(\mathrm{Jy})\end{array}$ & $\begin{array}{c}V_{\mathrm{LSR}} \\
\left(\mathrm{km} \mathrm{s}^{-1}\right)\end{array}$ & $\begin{array}{l}\text { FWHM } \\
\left(\mathrm{km} \mathrm{s}^{-1}\right)\end{array}$ \\
\hline $18517+0437$ (a) & $18: 54: 14.74$ & $+04: 41: 42.6$ & $2.22 \pm 0.05$ & 2.98 & 43.4 & 1.2 \\
\hline $18517+0437(b)$ & $18: 54: 14.46$ & $+04: 41: 44.6$ & $1.48 \pm 0.05$ & 1.60 & 43.9 & 1.4 \\
\hline $20126+4104$ (a) & $20: 14: 26.72$ & $+41: 13: 29.8$ & $1.40 \pm 0.01$ & 2.72 & -4.2 & 1.5 \\
\hline $20126+4104(b)$ & $20: 14: 25.24$ & $+41: 13: 34.9$ & $1.57 \pm 0.01$ & 1.46 & -2.1 & 2.2 \\
\hline $20126+4104(\mathrm{c})$ & $20: 14: 25.16$ & $+41: 13: 36.4$ & $1.79 \pm 0.01$ & 2.92 & -2.4 & 2.0 \\
\hline $20126+4104(d)$ & $20: 14: 25.44$ & $+41: 13: 37.3$ & $1.39 \pm 0.01$ & 6.14 & -2.9 & 1.4 \\
\hline $20126+4104(\mathrm{e})$ & $20: 14: 25.41$ & $+41: 13: 37.9$ & $1.49 \pm 0.01$ & 6.35 & -2.9 & 1.3 \\
\hline $23033+5951$ & $23: 05: 24.59$ & +60:08:09.4 & $0.16 \pm 0.04$ & 4.55 & -54.3 & 1.4 \\
\hline $23139+5939$ (a) & $23: 16: 10.86$ & $+59: 55: 20.7$ & $4.07 \pm 0.13$ & 0.44 & -44.6 & 1.7 \\
\hline $23139+5939(b)$ & $23: 16: 10.40$ & $+59: 55: 28.0$ & $0.33 \pm 0.13$ & 1.23 & -45.9 & 1.8 \\
\hline $23151+5912$ & $23: 17: 21.90$ & $+59: 28: 45.7$ & $4.30 \pm 0.22$ & 1.87 & -52.8 & 1.4 \\
\hline
\end{tabular}

Notes.

${ }^{a}$ Projected separation between $95 \mathrm{GHz} \mathrm{CH} 3 \mathrm{OH}$ maser position and nearest $3 \mathrm{~mm}$ continuum peak. Uncertainties estimated from signal to noise of the maser and continuum detections.

continuum emission to within 0'.6 (or $<2000-3000 \mathrm{AU}$ ), while the other nine masers are separated by $\sim 10^{4} \mathrm{AU}$ from the $3 \mathrm{~mm}$ continuum peaks (see Table 4). The projected spatial offsets are consistent with the expectation that the $95 \mathrm{GHz}$ masers are collisionally pumped at the interface between outflows and the interstellar medium (Minier \& Booth 2002). All of the $\mathrm{CH}_{3} \mathrm{OH}$ lines are found within $2 \mathrm{~km} \mathrm{~s}^{-1}$ of the systemic velocity of the HMPO, implying that the masers form in the colliding gas. Our finding that the maser line is found at nearly the same velocity as the ambient medium is consistent with the results of Minier \& Booth (2002), who find that this maser line is often, but not always, at the systemic velocity of the HMPO.

The location of known centimeter continuum sources, $\mathrm{H}_{2} \mathrm{O}$ masers, and $\mathrm{CH}_{3} \mathrm{OH}$ masers are marked on the contour maps in Figures 1 and 2. Six of the seven CARMA $3 \mathrm{~mm}$ continuum detections (all except IRAS 18517+0437) were included in an

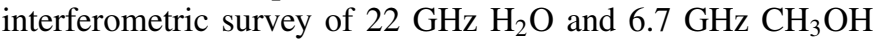
masers by Beuther et al. (2002b). Water masers have been identified toward all six cores detected with CARMA, and three of these cores also have $6.7 \mathrm{GHz} \mathrm{CH}_{3} \mathrm{OH}$ masers. For HMPOs with both $22 \mathrm{GHz} \mathrm{H}_{2} \mathrm{O}$ and $6.7 \mathrm{GHz} \mathrm{CH}_{3} \mathrm{OH}$ masers, the two types of masers are spatially coincident to within $1^{\prime \prime}-$ $2^{\prime \prime}$. Both types of masers also fall within $\sim 1^{\prime \prime}$ of our $3 \mathrm{~mm}$ continuum positions, as shown in Figure 1. Of the multiple
$3 \mathrm{~mm}$ components resolved in IRAS $19217+1651$ and IRAS $23033+5951$, one is coincident with $\mathrm{H}_{2} \mathrm{O}$ masers in each of the HMPOs.

\subsection{Radio Continuum}

Four of $103 \mathrm{~mm}$ continuum sources are located within $1^{\prime \prime}$ of a $3.6 \mathrm{~cm}$ radio continuum source. The $3 \mathrm{~mm}$ continuum emission for these objects may originate from free-free or thermal dust emission. If we assume that the sources in our sample are typical of other UCH II regions, the free-free emission will be optically thick (i.e., $S_{v} \propto v^{2}$ ) for $v<10-15 \mathrm{GHz}$, and optically thin $\left(S_{v} \propto v^{-0.1}\right)$ for higher frequencies (Hunter et al. 1998; Turner $\&$ Beck 2004; Zapata et al. 2008; Hunter et al. 2008). For a "typical" UCH II radio continuum spectrum, the expected $3 \mathrm{~mm}$ continuum flux density from free-free emission will be less than a factor of 2.7 higher than the $3.6 \mathrm{~cm}$ flux density. We estimate the relative contributions of free-free and dust emission to the observed $3 \mathrm{~mm}$ flux density using available data from the literature.

The source IRAS $19217+1651$ was observed with the VLA by Garay et al. (2007), who measured a $7 \mathrm{~mm}$ flux density of $50.4 \pm 0.16$ mJy toward the brighter of the two CARMA detections at $3 \mathrm{~mm}$. The $3 \mathrm{~mm}$ flux density of the brighter 

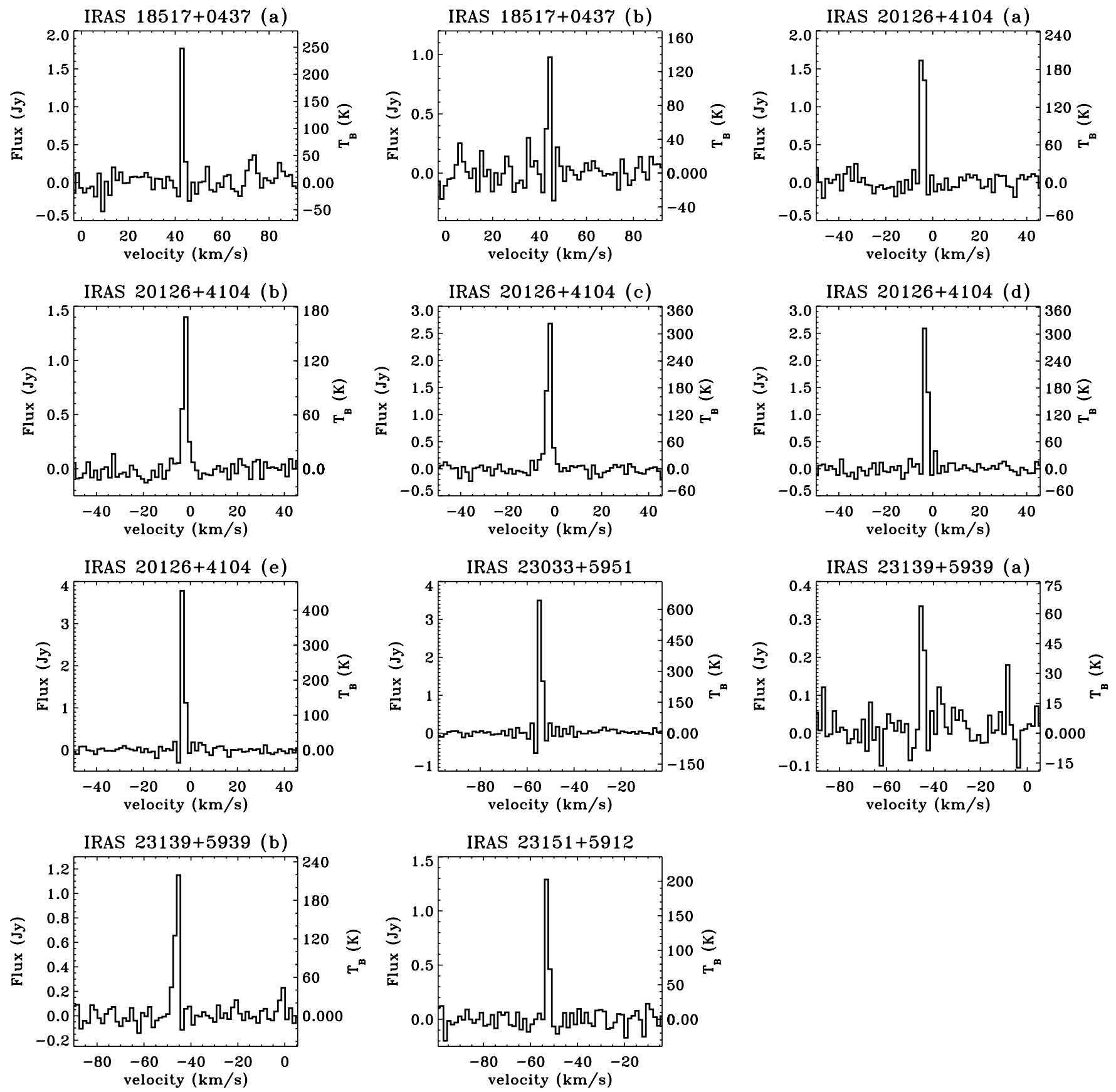

Figure 3. Spectra of the $95 \mathrm{GHz} \mathrm{CH} 3 \mathrm{OH}$ maser detections, with the same identifications shown in Figure 1 and Table 4.

component is $66 \pm 1 \mathrm{mJy}$; an additional calibration uncertainty of $\sim 10 \%$ should be included in both the $7 \mathrm{~mm}$ and $3 \mathrm{~mm}$ flux density measurements. The $7 \mathrm{~mm}$ detection is consistent with optically thin free-free emission (Garay et al. 2007), and implies that the free-free contribution to the $3 \mathrm{~mm}$ flux density is $\sim 46 \mathrm{mJy}$. The HMPO IRAS 20126+4104 was observed with the VLA between $20 \mathrm{~cm}$ and $7 \mathrm{~mm}$ by Hofner et al. (2007), who conclude that free-free emission should contribute $\sim 4 \mathrm{mJy}$ at $3 \mathrm{~mm}$, which is a small fraction of the $33 \pm 2 \mathrm{mJy}$ detected with CARMA. Our CARMA observations detect three regions of $3 \mathrm{~mm}$ emission toward the HMPO IRAS 23033+5951, of which the northernmost is associated with $3.6 \mathrm{~cm}$ emission of 1.7 mJy (Sridharan et al. 2002). For a "typical" UCH II radio continuum spectrum, the free-free emission would contribute less than $4.6 \mathrm{mJy}$ of the $8 \pm 1 \mathrm{mJy}$ detected with CARMA.
The HMPO IRAS $23139+5939$ has a flux density of $1.4 \mathrm{mJy}$ at $3.6 \mathrm{~cm}$ (Sridharan et al. 2002), so we assume that the free-free emission contributes no more than $3.8 \mathrm{mJy}$ of the $12 \pm 2 \mathrm{mJy}$ detected with CARMA at $3 \mathrm{~mm}$. For regions without $3.6 \mathrm{~cm}$ emission, we assume that dust accounts for all of the observed $3 \mathrm{~mm}$ flux density.

\subsection{Masses}

We derive the total (gas and dust) mass for each of the CARMA continuum detections from the observed $3 \mathrm{~mm}$ flux density, assuming optically thin emission from isothermal dust, using the equation

$$
M=\frac{S_{v} f_{d} D^{2}}{\kappa_{v} B_{v}(T)},
$$


where $S_{v}$ is the integrated flux density, $f_{d}$ is the fraction of $3 \mathrm{~mm}$ emission from dust, $D$ is the distance, $\kappa_{v}$ is the dust emissivity, and $B_{v}(T)$ is the Planck function. The value of the dust emissivity is extrapolated from a value of $\kappa_{0}=0.1 \mathrm{~cm}^{2}$ $\mathrm{g}^{-1}$ at $250 \mu \mathrm{m}$ assuming a power-law spectral index of $\beta=2$ (Hildebrand 1983; i.e., $\kappa_{v}=\kappa_{\mathrm{o}}\left(v / \nu_{\mathrm{o}}\right)^{\beta}$ ). For $95 \mathrm{GHz}$ continuum observations, the mass can be derived as

$$
\frac{M}{M_{\odot}}=0.55\left(\frac{S_{v} f_{d}}{\mathrm{mJy}}\right)\left(\frac{D}{\mathrm{kpc}}\right)^{2}\left(\frac{T}{50 \mathrm{~K}}\right)^{-1},
$$

where we use the Rayleigh-Jeans approximation to the Planck function and assume a temperature of $50 \mathrm{~K}$. Since the $3 \mathrm{~mm}$ continuum emitting regions are not resolved by our observations, a lower limit on the volume density is derived assuming spherical geometry with a diameter equal to the resolution and a mean molecular weight per free particle of 2.37. Estimated masses and densities for the individual $3 \mathrm{~mm}$ continuum sources are summarized in Table 3. The dominant source of uncertainty in the derived masses is the dust emissivity, which is uncertain by a factor of a few (Hildebrand 1983).

The masses of the sources detected in the CARMA $3 \mathrm{~mm}$ continuum maps range between 21 and $1200 M_{\odot}$ with volume densities in excess of $10^{8} \mathrm{~cm}^{-3}$. These masses and densities can be compared to the large-scale core traced by single-dish $1.2 \mathrm{~mm}$ continuum maps (Beuther et al. 2002a). Assuming that the large-scale core and the compact clumps have the same temperature and dust emissivity, we find that the compact $3 \mathrm{~mm}$ sources contain $\sim 20 \%$ of the total core mass, and have volume densities that are larger by a factor of $\sim 1000$ than the average core density.

\subsection{Outflows}

All 14 HMPO candidates in our sample were observed by Sridharan et al. (2002) with the CSO or IRAM $30 \mathrm{~m}$ telescopes to measure the $\mathrm{CO}(2-1)$ line profile. All were seen to have broad-line wings consistent with bipolar outflows, suggesting that outflow activity is common in these high-mass star-forming regions. The Sridharan et al. (2002) survey also searched for $\mathrm{SiO}(2-1)$ emission, another common outflow tracer, from 12 of the 14 HMPO candidates in our sample (all except IRAS 18517+0437 and IRAS 20332+4124), resulting in seven detections. Of the seven HMPO candidates with $\mathrm{SiO}(2-1)$ emission, six are associated with $3 \mathrm{~mm}$ continuum emission detected by CARMA, and none of the HMPO candidates without $\mathrm{SiO}(2-1)$ emission were detected with CARMA. Furthermore, of the five HMPO candidates that have nearby $95 \mathrm{GHz} \mathrm{CH}_{3} \mathrm{OH}$ maser emission, four are also associated with $\mathrm{SiO}(2-1)$ emission and the fifth was not included in the survey of Sridharan et al. (2002). However, the resolution of the single-dish observations $\left(10^{\prime \prime}-30^{\prime \prime}\right)$ is not sufficient to determine whether or not the HMPOs detected with CARMA are driving any these outflows.

Interferometric observations have reported detections of outflows from five of the 14 HMPOs in our CARMA survey.

1. Zhang et al. (2007) observed $\mathrm{SiO}(2-1)$ at $5^{\prime \prime}$ resolution toward the HMPO IRAS 18566+0408, and found that the emission traces a collimated outflow, originating from the position of the CARMA $3 \mathrm{~mm}$ continuum detection.

2. $\mathrm{SiO}(2-1)$ and $\mathrm{CO}(2-1)$ emission from the HMPO IRAS $19217+1651$ were observed by Beuther et al. (2004) at $2^{\prime \prime}-6 "$ resolution. There is a bipolar outflow seen in both transitions, originating from the position of the CARMA 3 mm continuum source.

3. IRAS $20126+4104$ has been observed at high resolution by Shepherd et al. (2000), who report the presence of a north-south oriented molecular outflow and a northwestsoutheast oriented jet centered on the position of the CARMA continuum source. Shocks identified by $\mathrm{H}_{2}$ and [S II] emission trace the jet, and are coincident with the positions of the $95 \mathrm{GHz} \mathrm{CH}_{3} \mathrm{OH}$ masers detected by CARMA, in agreement with the hypothesis that these maser lines are collisionally pumped at the interface between jets/ outflows and the ambient medium around the protostar.

4. CARMA found three $3 \mathrm{~mm}$ continuum peaks toward IRAS $23033+5951$, one associated with $3.6 \mathrm{~cm}$ emission, one with $\mathrm{a}_{2} \mathrm{O}$ maser, and one with $95 \mathrm{GHz} \mathrm{CH}_{3} \mathrm{OH}$ maser emission. This region has been studied by Reid \& Matthews (2008), who study the outflows in this region at $\sim 6^{\prime \prime}$ resolution. There are at least two outflows in this region, one identified by $\mathrm{HCO}^{+}(1-0)$ emission, and one identified by $\mathrm{CH}_{3} \mathrm{OH}(2-$ 1) and $\mathrm{SiO}(2-1)$ emission. The CARMA $3 \mathrm{~mm}$ continuum source coincident with the $\mathrm{H}_{2} \mathrm{O}$ maser is most likely to be driving the $\mathrm{SiO}$ outflow, and it is unclear what drives the $\mathrm{HCO}^{+}$outflow or is exciting the $95 \mathrm{GHz} \mathrm{CH}_{3} \mathrm{OH}$ maser.

5. The $3 \mathrm{~mm}$ continuum source detected with CARMA in IRAS 23151+5912 is coincident with "Peak 1" in the $875 \mu \mathrm{m}$ continuum map of Beuther et al. (2007) and with the $3.4 \mathrm{~mm}$ and $1.3 \mathrm{~mm}$ continuum source detected by Qiu et al. (2007). There are at least two outflows, identified by $\mathrm{SiO}(2-1)$ and (8-7) emission, in this region, one oriented roughly west-east and the other north-south (Beuther et al. 2007; Qiu et al. 2007). The outflows are likely driven by two protostars, and it is not clear if the $3 \mathrm{~mm}$ continuum emission detected with CARMA corresponds to one or both protostars, or if the $95 \mathrm{GHz} \mathrm{CH} \mathrm{CH}_{3} \mathrm{OH}$ maser is related to the interaction between the west-east outflow and the ambient medium.

\section{DISCUSSION}

One proposed evolutionary sequence for HMPOs is that they originate from low or intermediate mass protostars that are accreting material from the surrounding core, presumably via a circumstellar disk (Beuther et al. 2007). As the protostar increases in mass, an $\mathrm{H}$ II region ultimately forms that eventually expands and halts accretion. While it is unclear if $\mathrm{H}_{2} \mathrm{O}$ or $6.7 \mathrm{GHz} \mathrm{CH}_{3} \mathrm{OH}$ masers originate from the disk or outflow, the observational evidence suggests these masers trace an early stage of evolution before the $\mathrm{H}$ II region has expanded significantly (Lo et al. 1975; Genzel \& Downes 1977; Walsh et al. 1998; Codella et al. 2004). In this scenario, a young HMPO with an accretion disk would be identified by its water and/or methanol masers and millimeter wavelength thermal emission from the dust around the protostar. When the HMPO is "intermediate aged," an UCH II region detectable with centimeter wavelength continuum emission would grow but will not immediately disrupt the accretion, so masers and dust thermal emission would also be present. As the UCH II region expands, it will destroy the disk and cutoff the masers. We now evaluate whether or not the CARMA observations are consistent with this evolutionary sequence.

First, we discuss if the compact sources detected in the CARMA $3 \mathrm{~mm}$ continuum maps signify the presence of a highmass protostar. The masses of the compact sources range between 21 and $1200 M_{\odot}$ contained within a region $\lesssim 10,000 \mathrm{AU}$ 
in diameter. Thus these compact $3 \mathrm{~mm}$ continuum sources are substantially denser and more massive than the dense cores found in nearby clouds known to be forming low-mass stars. Even if we assume only $10 \%$ of the core mass forms into a star, a mid B-type star can potentially form in these cores. Moreover, four of the $3 \mathrm{~mm}$ continuum sources are spatially coincident with compact $\mathrm{H}$ II regions, confirming directly the presence of a high-mass star. Therefore, we assume that all of the compact $3 \mathrm{~mm}$ continuum sources represent dense dust and gas surrounding a high-mass star or protostar. We cannot determine if the material is distributed in a disk or compact envelope (e.g., Beltrán et al. 2004; Cesaroni et al. 2005; Reid \& Matthews 2008), however, as the dust continuum emission is unresolved by these observations.

Since $3 \mathrm{~mm}$ continuum emission is detected toward seven of the 14 HMPOs in our survey we investigate whether the $50 \%$ detection rate is set by physical differences between the highmass cores or by selection bias. A bias may be introduced since the HMPOs span a factor of $\sim 6$ in distance and a factor of $\sim 10$ in luminosity, but the sensitivity in each of the CARMA maps is approximately the same. In the top panel of Figure 4, we plot the noise in the CARMA $3 \mathrm{~mm}$ continuum maps as a function of the distance to the HMPO for sources with (black circles) and without (gray circles) CARMA continuum detections. No clear differences in the noise characteristics or source distances are found between the two samples. The middle panel shows the core masses derived from single-dish $1.2 \mathrm{~mm}$ continuum maps versus the bolometric luminosity of the core. Again, sources with and without $3 \mathrm{~mm}$ CARMA continuum detections share similar distributions, indicating that the CARMA observations did not preferentially detect cores with the highest mass or luminosity. Finally, the bottom panel in Figure 4 shows how the peak flux density in the MAMBO single-dish $1.2 \mathrm{~mm}$ continuum maps varies with the integrated $1.2 \mathrm{~mm}$ flux density. We detect (at the $\geqslant 3 \sigma$ level) $3 \mathrm{~mm}$ continuum emission from all seven of the HMPOs with $1.2 \mathrm{~mm}$ peak fluxes above $300 \mathrm{mJy} /\left(11^{\prime \prime}\right)^{2}$, while none of the seven HMPOs below that threshold are detected.

Is there an observational bias for detecting only those sources with the highest peak surface brightnesses at $1.2 \mathrm{~mm}$, or is there an intrinsic difference between those cores above and below the $300 \mathrm{mJy}$ beam $^{-1}$ threshold? The peak $1.2 \mathrm{~mm}$ flux densities in our sample are all $\geqslant 100 \mathrm{mJy}_{\text {beam }}{ }^{-1}$. If the $1.2 \mathrm{~mm}$ flux density in the $11^{\prime \prime}$ single-dish beam originates from an unresolved disk or envelope, we would expect to observe flux densities of $\geqslant 4 \mathrm{mJy}$ beam $^{-1}$ at $3 \mathrm{~mm}$ assuming an emissivity spectral index of $\beta=1$, which is appropriate for disks (Beckwith \& Sargent 1991). The noise in the $3 \mathrm{~mm}$ continuum maps is approximately $1 \mathrm{mJy}$ beam $^{-1}$, and all 14 HMPO candidates would be detectable at $\geqslant q 3 \sigma$ level if the central emission were compact.

Given that all 14 HMPO candidates could have been detected with CARMA, we consider whether differences in the CARMA $3 \mathrm{~mm}$ continuum detection rate versus peak $1.2 \mathrm{~mm}$ flux density could be explained by the presence or absence of an unresolved disk or envelope. The median integrated $3 \mathrm{~mm}$ flux density detected with CARMA is $10 \mathrm{mJy}$, which, assuming an emissivity spectral index of $\beta=1$, would contribute $\sim 180 \mathrm{mJy} \mathrm{beam}^{-1}$ of emission at $1.2 \mathrm{~mm}$. This is approximately equal to the difference between the peak $1.2 \mathrm{~mm}$ flux densities of the faintest HMPOs detected by CARMA ( 400 $\mathrm{mJy}^{\text {beam }}{ }^{-1}$ at $\left.1.2 \mathrm{~mm}\right)$ and the brightest HMPOs not detected with CARMA $(\sim 250 \mathrm{mJy}$ beam $\left.^{-1}\right)$. Thus the lack of CARMA detections around sources with low peak $1.2 \mathrm{~mm}$ flux densities, but otherwise large
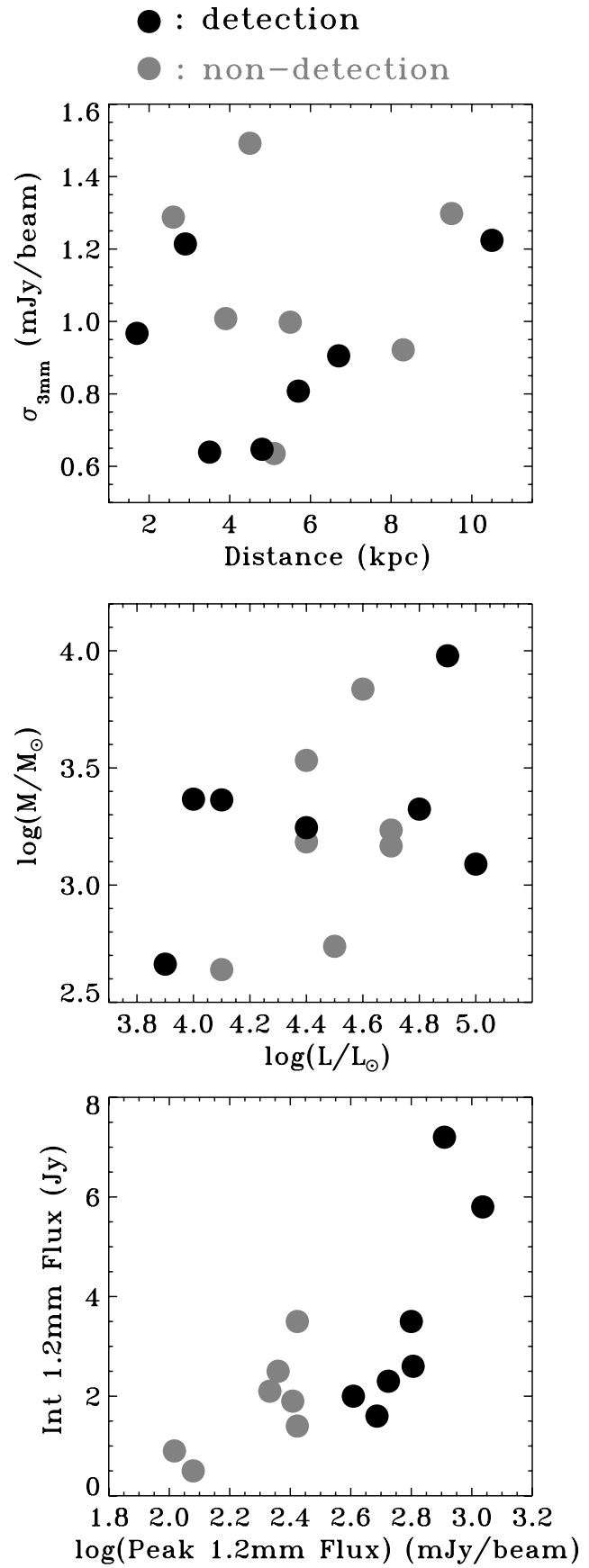

Figure 4. Properties of the HMPO candidates with (black circles) and without (gray circles) CARMA $3 \mathrm{~mm}$ continuum detections. Top: rms noise in the CARMA 3 mm image vs. the distance to the HMPO. Middle: mass of the dense core, as measured by $1.2 \mathrm{~mm}$ continuum images (Beuther et al. 2002a), vs. the bolometric luminosity. Bottom: integrated flux density in the $1.2 \mathrm{~mm}$ single-dish maps vs. the peak $1.2 \mathrm{~mm}$ flux density. These figures show that HMPOs with and without CARMA detections have similar noise characteristics, distances, core masses, and luminosities. However, sources without CARMA continuum detections tend to have lower peak flux densities. The data displayed in these plots are presented in Tables 1 and 2 .

integrated $1.2 \mathrm{~mm}$ flux densities and FIR luminosities, may indicate that a compact disk or envelope has dissipated around these sources.

Since the detection rate of $3 \mathrm{~mm}$ sources is not strongly biased by the range of distances, masses, and luminosities in our sample, we investigate if the evolutionary stage correlates with the detection rate of compact $3 \mathrm{~mm}$ sources. The signposts of the age sequence that we are testing, in order of increasing 
Table 5

Association of Masers and Continuum Detections

\begin{tabular}{|c|c|c|c|c|c|c|c|}
\hline IRAS Name & Group $^{a}$ & $3 \mathrm{~mm}$ Continuum ${ }^{\mathrm{b}}$ & $3.6 \mathrm{~cm}$ Continuum ${ }^{\mathrm{c}}$ & $\begin{array}{c}\mathrm{H}_{2} \mathrm{O} \text { Maser }^{\mathrm{c}} \\
22 \mathrm{GHz}\end{array}$ & $\begin{array}{c}\mathrm{CH}_{3} \mathrm{OH} \mathrm{Maser}{ }^{\mathrm{c}} \\
6.7 \mathrm{GHz}\end{array}$ & $\begin{array}{c}\mathrm{CH}_{3} \mathrm{OH} \text { Maser } \\
95 \mathrm{GHz}\end{array}$ & $\begin{array}{c}\text { OH Maser } \\
1.7 \mathrm{GHz}\end{array}$ \\
\hline 18345-0641 & 1 & $\mathrm{~N}$ & $\mathrm{~N}$ & Y & $\mathrm{Y}$ & $\mathrm{N}$ & $\mathrm{Y}$ \\
\hline $18440-0148$ & 1 & $\mathrm{~N}$ & $\mathrm{~N}$ & $\mathrm{~N}$ & $\mathrm{Y}$ & $\mathrm{N}$ & $\mathrm{Y}$ \\
\hline $18517+0437$ & 1 & $\mathrm{Y}$ & $\mathrm{N}$ & $\mathrm{Y}$ & $\mathrm{Y}$ & $\mathrm{Y}$ & $\mathrm{N}$ \\
\hline $18566+0408$ & 1 & $\mathrm{Y}$ & $\mathrm{N}$ & $\mathrm{Y}$ & $\mathrm{Y}$ & $\mathrm{N}$ & $\mathrm{Y}$ \\
\hline $23151+5912$ & 1 & $\mathrm{Y}$ & $\mathrm{N}$ & $\mathrm{Y}$ & $\mathrm{N}$ & $\mathrm{Y}$ & $\mathrm{N}$ \\
\hline $19217+1651$ & 2 & $\mathrm{Y}$ & $\mathrm{Y}$ & $\mathrm{Y}$ & $\mathrm{Y}$ & $\mathrm{N}$ & $\mathrm{Y}$ \\
\hline $20126+4104$ & 2 & $\mathrm{Y}$ & $\mathrm{Y}$ & $\mathrm{Y}$ & $\mathrm{Y}$ & $\mathrm{Y}$ & $\mathrm{Y}$ \\
\hline $20332+4124$ & 2 & $\mathrm{~N}$ & $\mathrm{Y}$ & $\mathrm{Y}$ & $\mathrm{N}$ & $\mathrm{N}$ & $\mathrm{N}$ \\
\hline $23033+5951$ & 2 & $\mathrm{Y}$ & $\mathrm{Y}$ & $\mathrm{Y}$ & $\mathrm{N}$ & $\mathrm{Y}$ & $\mathrm{N}$ \\
\hline $23139+5939$ & 2 & $\mathrm{Y}$ & $\mathrm{Y}$ & $\mathrm{Y}$ & $\mathrm{Y}$ & $\mathrm{Y}$ & $\mathrm{Y}$ \\
\hline $19220+1432$ & 3 & $\mathrm{~N}$ & $\mathrm{Y}$ & $\mathrm{N}$ & $\mathrm{N}$ & $\mathrm{N}$ & $\mathrm{Y}$ \\
\hline $20205+3948$ & 3 & $\mathrm{~N}$ & $\mathrm{Y}$ & $\mathrm{N}$ & $\mathrm{N}$ & $\mathrm{N}$ & $\mathrm{N}$ \\
\hline $22134+5834$ & 3 & $\mathrm{~N}$ & $\mathrm{Y}$ & $\mathrm{N}$ & $\mathrm{N}$ & $\mathrm{N}$ & $\mathrm{N}$ \\
\hline $22570+5912$ & 3 & $\mathrm{~N}$ & $\mathrm{Y}$ & $\mathrm{N}$ & $\mathrm{N}$ & $\mathrm{N}$ & $\mathrm{N}$ \\
\hline
\end{tabular}

Notes.

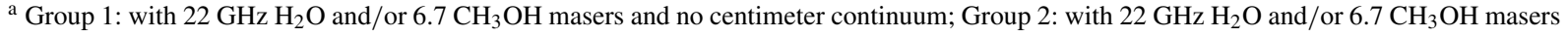
and centimeter continuum; Group 3: without $22 \mathrm{GHz} \mathrm{H}_{2} \mathrm{O}$ or $6.7 \mathrm{CH}_{3} \mathrm{OH}$ masers and with centimeter continuum.

b This study.

c Sridharan et al. (2002).

${ }^{\mathrm{d}}$ Edris et al. (2007).

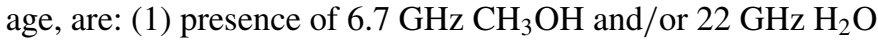
maser emission and no centimeter continuum emission, (2) both maser and centimeter continuum emission, and (3) centimeter continuum emission but an absence of masers (Lo et al. 1975; Genzel \& Downes 1977; Codella et al. 1994; Codella \& Felli 1995). For each of the 14 massive cores in our survey, we list the presence of $3 \mathrm{~mm}$ continuum, centimeter continuum, and maser emission in Table 5.

We detect $3 \mathrm{~mm}$ continuum emission from $3 / 5$ of the "youngest" HMPOs, $4 / 5$ of the "intermediate" aged HMPOs and $0 / 4$ of the "oldest" HMPOs. Thus, the $3 \mathrm{~mm}$ continuum emission is preferentially detected toward the sources with maser emission, consistent with the proposed evolutionary sequence for high-mass stars. Further, we would expect that the $\mathrm{H}_{2} \mathrm{O}$ masers and the $6.7 \mathrm{GHz} \mathrm{CH}_{3} \mathrm{OH}$ masers to be nearly coincident with the compact $3 \mathrm{~mm}$ sources, since very long baseline interferometry (VLBI) observations suggest that these masers originate from shocks associated with winds or jets near the base of the molecular outflow (Goddi et al. 2005; Moscadelli et al. 2005). All $13 \mathrm{H}_{2} \mathrm{O}$ masers with interferometric positions, and five of the eight $6.7 \mathrm{GHz} \mathrm{CH}_{3} \mathrm{OH}$ masers, are in fact located within 1 1.5 of a compact $3 \mathrm{~mm}$ continuum source. It is possible that the $\mathrm{CH}_{3} \mathrm{OH}$ maser sources without associated $3 \mathrm{~mm}$ continuum emission may have too low of disk or envelope mass to be detected in our observations.

In summary, all of the $\mathrm{H}_{2} \mathrm{O}$ and most of the $6.7 \mathrm{GHz}$ $\mathrm{CH}_{3} \mathrm{OH}$ maser emission appear to be related to massive disks or envelopes, but these disks/envelopes are not present toward sources that have radio continuum emission but no maser emission. The probability of association between HMPOs with $3 \mathrm{~mm}$ continuum emission and HMPOs with $22 \mathrm{GHz}_{2} \mathrm{O}$ masers and $6.7 \mathrm{GHz} \mathrm{CH}_{3} \mathrm{OH}$ masers is $98 \%$ and $71 \%$, respectively. These $3 \mathrm{~mm}$ continuum sources are also likely to be driving outflows, and there is a $98 \%$ likelihood that $3 \mathrm{~mm}$ continuum emission is associated with two possible outflow tracers, $\mathrm{SiO}(2-1)$ emission and $95 \mathrm{GHz} \mathrm{CH} \mathrm{CH}_{3} \mathrm{OH}$ masers. There is a $99.8 \%$ likelihood of association between these two spectral lines. These observa- tions are consistent with the hypothesis that as an $\mathrm{H}$ II region around a high-mass protostar expands, it destroys the surrounding disk and envelope, and consequently shuts off the source of the outflows and $\mathrm{H}_{2} \mathrm{O}$ and $\mathrm{CH}_{3} \mathrm{OH}$ masers.

\section{SUMMARY}

In this paper, we tested the hypothesis that $\mathrm{H}_{2} \mathrm{O}$ and $\mathrm{CH}_{3} \mathrm{OH}$ masers are signposts of an early phase in the evolution of a high-mass protostellar object before an expanding UCH II region has destroyed the accretion disk. We used CARMA to observe the $3 \mathrm{~mm}$ continuum emission at $1^{\prime \prime}$ resolution around a sample of HMPOs with and without masers and centimeter continuum emission. We also observed the $95 \mathrm{GHz} \mathrm{CH}_{3} \mathrm{OH}$ line to determine its association with the thermal emission from dust surrounding the protostars.

We detect (at $\geqslant 3 \sigma$ significance) $3 \mathrm{~mm}$ continuum emission from all seven HMPOs with MAMBO $1.2 \mathrm{~mm}$ continuum peak fluxes (taken from Beuther et al. 2002a) above $300 \mathrm{mJy} /\left(11^{\prime \prime}\right)^{2}$, while none of the seven HMPOs with peak fluxes below this threshold were detected in our survey. We argue that this is not merely an observational selection effect, because all 14 HMPO candidates could have been detected in the CARMA maps if their $1.2 \mathrm{~mm}$ peak fluxes originated from disks or compact envelopes, and were therefore concentrated into the $\sim 1^{\prime \prime}$ CARMA beam. The detection rate of HMPOs at $3 \mathrm{~mm}$ is not correlated with distance to the HMPO, the $1.2 \mathrm{~mm}$ integrated flux detected with MAMBO, the total luminosity of the HMPO or its mass (see Figure 4).

All seven HMPOs detected at $3 \mathrm{~mm}$ are associated with $\mathrm{H}_{2} \mathrm{O}$ masers, and only two of the seven HMPOs not detected at $3 \mathrm{~mm}$ are associated with $\mathrm{H}_{2} \mathrm{O}$ masers. The $22 \mathrm{GHz} \mathrm{H}_{2} \mathrm{O}$ masers and $95 \mathrm{GHz} \mathrm{CH}_{3} \mathrm{OH}$ lines are significantly ( $98 \%$ probability) associated with the detection rate of $3 \mathrm{~mm}$ continuum emission. There is no significant association between the detection rate of $3 \mathrm{~mm}$ continuum and $6.7 \mathrm{GHz} \mathrm{CH} 3 \mathrm{OH}$ or $1.7 \mathrm{GHz} \mathrm{OH}$ masers 
or centimeter continuum emission. The presence of $\mathrm{SiO}$ (21) emission in the study of Sridharan et al. (2002) was also a useful predictor of CARMA $3 \mathrm{~mm}$ continuum detections $(98 \%$ probability) and $95 \mathrm{GHz} \mathrm{CH}_{3} \mathrm{OH}$ masers (99.8\% probability).

The general picture of the evolution of high-mass protostars is consistent with our observations that the $3 \mathrm{~mm}$ continuum emission comes from a disk and/or compact envelope around an HMPO. Our observations suggest that $\mathrm{H}_{2} \mathrm{O}$ masers are associated with these disks/envelopes, and that they are present both prior to and during the formation of an UCH II region. When the UCH II region disrupts the disk and compact envelope around the $\mathrm{HMPO}$, the $\mathrm{H}_{2} \mathrm{O}$ and $95 \mathrm{GHz} \mathrm{CH}_{3} \mathrm{OH}$ masers are likely to be cutoff and the $3 \mathrm{~mm}$ continuum emission falls below our threshold of detectability.

We thank the referee for improving the clarity and increasing the scope of this paper. We thank the CARMA staff, students, and postdocs for their help in making these observations. We acknowledge support from the Owens Valley Radio Observatory, which is supported by the National Science Foundation through grant AST 05-40399. Support for CARMA construction was derived from the Gordon and Betty Moore Foundation, the Kenneth T. and Eileen L. Norris Foundation, the Associates of the California Institute of Technology, the states of California, Illinois and Maryland, and the National Science Foundation. Ongoing CARMA development and operations are supported by the National Science Foundation under a cooperative agreement, and by the CARMA partner universities. This research has made use of the SIMBAD database, operated at CDS, Strasbourg, France.

\section{REFERENCES}

Altenhoff, W. J., Downes, D., Pauls, T., \& Schraml, J. 1979, A\&AS, 35, 23 Beckwith, S. V. W., \& Sargent, A. I. 1991, ApJ, 381, 250

Beltrán, M. T., Cesaroni, R., Neri, R., Codella, C., Furuya, R. S., Testi, L., \& Olmi, L. 2004, ApJ, 601, L187

Benjamin, R. A., et al. 2003, PASP, 115, 953

Beuther, H., Schilke, P., \& Gueth, F. 2004, ApJ, 608, 330

Beuther, H., Schilke, P., Menten, K. M., Motte, F., Sridharan, T. K., \& Wyrowski, F. 2002a, ApJ, 566, 945

Beuther, H., Walsh, A., Schilke, P., Sridharan, T. K., Menten, K. M., \& Wyrowski, F. 2002b, A\&A, 390, 289

Beuther, H., Zhang, Q., Hunter, T. R., Sridharan, T. K., \& Bergin, E. A. 2007, A\&A, 473, 493

Bronfman, L., Nyman, L.-A., \& May, J. 1996, A\&AS, 115, 81

Caratti O Garatti, A., Froebrich, D., Eislöffel, J., Giannini, T., \& Nisini, B. 2008, A\&A, 485, 137

Cesaroni, R., Neri, R., Olmi, L., Testi, L., Walmsley, C. M., \& Hofner, P. 2005, A\&A, 434, 1039

Codella, C., \& Felli, M. 1995, A\&A, 302, 521

Codella, C., Felli, M., Natale, V., Palagi, F., \& Palla, F. 1994, A\&A, 291, 261

Codella, C., Lorenzani, A., Gallego, A. T., Cesaroni, R., \& Moscadelli, L. 2004, A\&A, 417, 615

Cyganowski, C. J., et al. 2008, AJ, 136, 2391

De Buizer, J. M. 2003, MNRAS, 341, 277

Edris, K. A., Fuller, G. A., \& Cohen, R. J. 2007, A\&A, 465, 865
Edris, K. A., Fuller, G. A., Cohen, R. J., \& Etoka, S. 2005, A\&A, 434, 213 Egan, M. P., Shipman, R. F., Price, S. D., Carey, S. J., Clark, F. O., \& Cohen, M. 1998, ApJ, 494, L199

Garay, G., \& Lizano, S. 1999, PASP, 763, 1049

Garay, G., Rodríguez, L. F., \& de Gregorio-Monsalvo, I. 2007, AJ, 134, 906 Genzel, R., \& Downes, D. 1977, A\&AS, 30, 145

Goddi, C., Moscadelli, L., Alef, W., Tarchi, A., Brand, J., \& Pani, M. 2005, A\&A, 432, 161

Gregory, P. C., \& Condon, J. J. 1991, ApJS, 75, 1011

Griffith, M. R., Wright, A. E., Burke, B. F., \& Ekers, R. D. 1994, ApJS, 90, 179

Haynes, R. F., Caswell, J. L., \& Simons, L. W. J. 1979, Aust. J. Phys Astrophys. Suppl., 48, 1

Hildebrand, R. H. 1983, QJRAS, 24, 267

Hofner, P., Cesaroni, R., Olmi, L., Rodríguez, L. F., Martí, J., \& Araya, E. 2007, A\&A, 465, 197

Hunter, T. R., Brogan, C. L., Indebetouw, R., \& Cyganowski, C. J. 2008, ApJ, 680,1271

Hunter, T. R., Neugebauer, G., Benford, D. J., Matthews, K., Lis, D. C., Serabyn, E., \& Phillips, T. G. 1998, ApJ, 493, L97

Keto, E. 2002, ApJ, 580, 980

Keto, E. 2007, ApJ, 666, 976

Kumar, M. S. N., \& Grave, J. M. C. 2007, A\&A, 472, 155

Lo, K. Y., Burke, B. F., \& Haschick, A. D. 1975, ApJ, 202, 81

Maršálková, P. 1974, Ap\&SS, 27, 3

McKee, C. F., \& Ostriker, E. C. 2007, ARA\&A, 45, 565

Menten, K. M., Pillai, T., \& Wyrowski, F. 2005, in IAU Symp. 227, Massive Star Birth: A Crossroads of Astrophysics, ed. R. Cesaroni, R. M. R. Felli, E. Churchwell, \& M. Walmsley (Cambridge: Cambridge Univ. Press), 23

Minier, V., \& Booth, R. S. 2002, A\&A, 387, 179

Minier, V., Burton, M. G., Hill, T., Pestalozzi, M. R., Purcell, C. R., Garay, G., Walsh, A. J., \& Longmore, S. 2005, A\&A, 429, 945

Molinari, S., Brand, J., Cesaroni, R., \& Palla, F. 1996, A\&A, 308, 573

Molinari, S., Testi, L., Rodríguez, L. F., \& Zhang, Q. 2002, ApJ, 570, 758

Moscadelli, L., Cesaroni, R., \& Rioja, M. J. 2005, A\&A, 438, 889

Pandian, J. D., Goldsmith, P. F., \& Deshpande, A. A. 2007, ApJ, 656, 255

Perault, M., et al. 1996, A\&A, 315, L165

Qiu, K., Zhang, Q., Beuther, H., \& Yang, J. 2007, ApJ, 654, 361

Rathborne, J. M., Jackson, J. M., \& Simon, R. 2006, ApJ, 641, 389

Rathborne, J. M., Simon, R., \& Jackson, J. M. 2007, ApJ, 662, 1082

Reid, M. A., \& Matthews, B. C. 2008, ApJ, 675, 1343

Sault, R. J., Teuben, P. J., \& Wright, M. C. H. 1995, in ASP Conf. Ser. 77, Astronomical Data Analysis Software and Systems IV, ed. R. A. Shaw, H. E. Payne, \& J. J. E. Hayes (San Francisco, CA: ASP), 433

Shepherd, D. S., Yu, K. C., Bally, J., \& Testi, L. 2000, ApJ, 535, 833

Sridharan, T. K., Beuther, H., Schilke, P., Menten, K. M., \& Wyrowski, F. 2002, ApJ, 566, 931

Szymczak, M., Hrynek, G., \& Kus, A. J. 2000, A\&AS, 143, 269

Torrelles, J. M., et al. 2003, ApJ, 598, L115

Turner, J. L., \& Beck, S. C. 2004, ApJ, 602, L85

van der tak, F. F. S., \& Menten, K. M. 2005, A\&A, 2005, 437, 947

Walmsley, M. 1995, RevMexAA, 1, 137

Walsh, A. J., Burton, M. G., Hyland, A. R., \& Robinson, G. 1998, MNRAS, 301,640

Wang, Y., Zhang, Q., Rathborne, J. M., Jackson, J., \& Wu, Y. 2006, ApJ, 651, L125

Wood, D. O. S., \& Churchwell, E. 1989, ApJ, 340, 265

Wright, A. E., Griffith, M. R., Burke, B. F., \& Ekers, R. D. 1994, ApJS, 91, 111

Zapata, L. A., Leurini, S., Menten, K. M., Schilke, P., Rolffs, R., \& Hieret, C. 2008, AJ, 136, 1455

Zhang, Q., Hunter, T. R., Brand, J., Sridharan, T. K., Molinari, S., Kramer, M. A., \& Cesaroni, R. 2001, ApJ, 552, L167

Zhang, Q., Sridharan, T. K., Hunter, T. R., Chen, Y., Beuther, H., \& Wyrowski, F. 2007, A\&A, 470, 269

Zinnecker, H., \& Yorke, H. W. 2007, ARA\&A, 45, 481 